\title{
Intramedullary Spinal Cord Compression Caused by Histoplasma capsulatum: A Case Report and Meta-Analysis
}

\author{
Dominique N'Dri-Oka ${ }^{1}$ Nicole Adou ${ }^{1}$ André Tokpa ${ }^{1}$ Louis Derou ${ }^{1}$ \\ ${ }^{1}$ Neurosurgery Department at Abidjan Yopougon University Hospital \\ in Ivory Coast, Abidjan, Ivory Coast \\ Address for correspondence Dominique N'dri Oka, MD, MSc, \\ Neurosurgery Department at Abidjan Yopougon University Hospital \\ in Ivory Coast, 21 BP 632 Abidjan 21, Ivory Coast \\ Indian J Neurosurg 2015;4:109-113. \\ (e-mail: ndriokad@gmail.com).
}

\begin{abstract}
Nine cases of intramedullar histoplasmosis have been published in the literature. In six cases, spinal cord compression was associated with brain localization or with contex of disseminated histoplasmosis. The authors are reporting here the third isolated spinal cord compression in immunocompetent 42-year-old African-rabbits breeder, a woman. This case was successfully managed with surgical removal of the lesion associated to itraconazole during 8 months. Intramedullar lesion because of the Histoplasma capsulatum was necrosis granulomatous localized at spinal conus.

In conclusion, according to literature data the most frequent spinal cord compression

Keywords

- Histoplasma capsulatum

- necrosis granuloma

- spinal cord lesion

- meta-analysis caused by histoplasmosis capsulatum. General risk factors include residence in endemic areas as well as immunosuppression. Endemic areas include Africa, Australia, parts of Eastern Asia, and America (Mississippi, Missouri, and Ohio River valleys). Initial localization was cutaneous. Two histopathological forms reported are abscess and necrotizing granuloma. Management is mainly based on antifungus like itraconazole. Surgery is only necessary for the etiology diagnosis.
\end{abstract}

\section{Introduction}

Histoplasmosis is an intracellular infection caused by inhalation of conidia from fungus Histoplasma capsulatum. It grows as a mycelium in soils enriched by organic nitrogen sources such as bird and bat droppings. Histoplasma capsulatum is a dimorphic fungus. ${ }^{1}$ This is seen in endemic areas including Africa, Australia, part of Eastern Asia, and America (Mississippi, Missouri, and Ohio River valleys, Mexico, north of Latin America as Brazil). ${ }^{2}$ General risk factors include residence in endemic areas as well as immunosuppression. Cutaneous localization is more frequent and characterized by granulomatous lesion. The other localizations often encountered are bone and lungs. Histoplasmosis caused by Histoplasma capsulatum is a benign and cosmopolitan condition whereas the African histoplasmosis observed in the African continent, mainly between the tropics of cancer and capricon., ${ }^{2,3}$ Even pulmonary inhalation or cutaneous direct inoculations are incriminated. The pathogenesis of spinal intramedullary histoplasmosis is still unknown while cerebral histoplasmosis frequent, intramedullary spinal compression by histoplasmosis is an extremely rare condition. Here, we reported one case of intramedullary spinal compression by Histoplasma capsulatum and discuss according to the literature review, its pathogenesis, diagnosis, and management.

\section{Materials and Methods}

Case report is followed by literature review. That literature review is made on Medline/PubMed, Scopus, Scholar Google, Elsevier, Cochrane library, and Embase databases. The keywords used to assess articles were as follows: received

December 21, 2014

accepted

May 25, 2015

published online

July 28, 2015
DOI http://dx.doi.org/

10.1055/s-0035-1558837. ISSN 2277-954X. (c) 2015 Neurological Surgeons' Society of India
License terms

(잉 (1) $\Theta \circledast$ 
intramedullary spinal cord/histoplasmosis/Histoplasma capsulatum, and histoplasmosis central nerve. The search conducted yielded results such as the articles by Bazan et $\mathrm{al}^{3}$ and Desai et al. ${ }^{4}$ The Portuguese article entitled "Myelopathy caused by histoplasmosis: report of a case" was not included because only meningeal was of interest. ${ }^{5}$

\section{Results}

\section{Case Report}

An immunocompetent 42-year-old woman was admitted to neurosurgical emergency, complaining of progressive paraparesis 2 weeks later. She worked as a rabbit breeder. Her antecedents were without features and she denied living in endemic regions. During her hospitalization, she complained of intermittent medullar claudication. Walking was difficult. A few days later, a total functional impairment of the lower limb settled very quickly. Physicians' examination demonstrated frank paraplegia with motor deficit at grade $1 / 5$ according to American motor score grading and sphincter disorders, absent tone, urinary incontinence, and decreased sensory from abdominal level caudally. Thoracolumbar magnetic resonance imaging (MRI) was conducted and revealed an enlargement on conus medullaris, heterogeneous enhancement of the cord, and meningeal enhancement on T1-MRI weighted with gadolinium sagittal.

-Fig. 1 shows intramedullary ring and - Fig. 2 shows enhancing lesion at T2. No cerebral MRI was performed. The

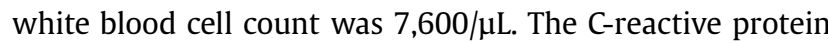
was $0.3 \mathrm{mg} / \mathrm{L}$. Human immunodeficiency virus (HIV) serology was negative.

The patient underwent laminectomy from T12 to L1. When the median longitudinal opening of the dura matter was performed, an enlargement of the spinal cord was observed. It also allowed highlighting harmoniously swollen spinal with outbreaks of blackish appearance within a pearly white tumor. Opening of the mass to a blackish substance issue evoke intratumoral hematoma. No pus was encountered.

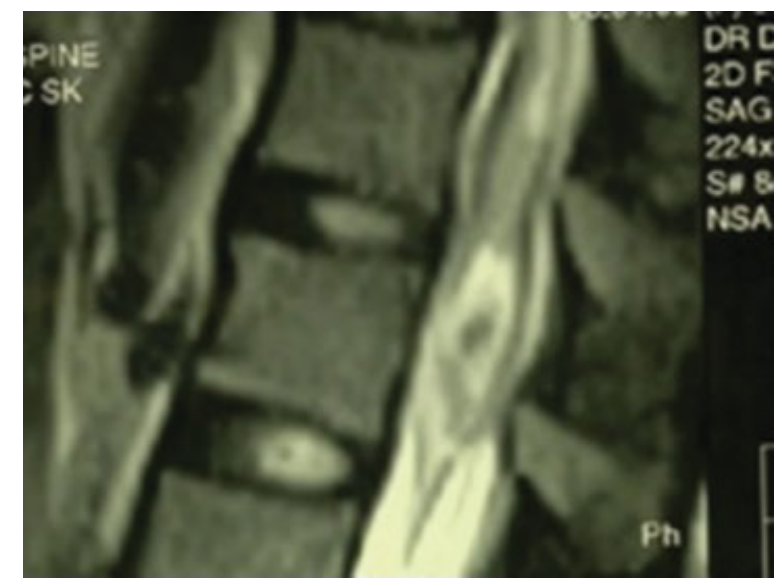

Fig. 1 Magnetic resonance image of the spinal cord T1-weighted sagittal image showing an enlargement of the conus heterogeneous enhancement.

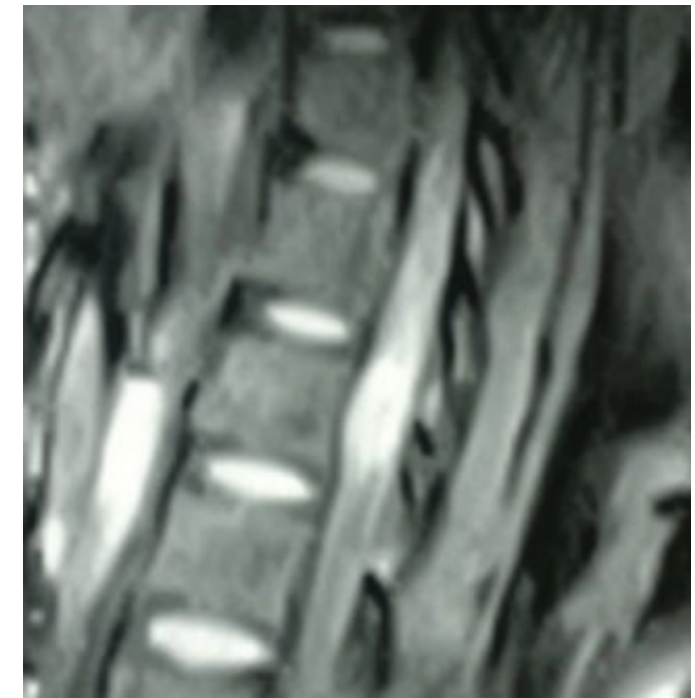

Fig. 2 Magnetic resonance image of the spinal cord T2-weighted sagittal image showing an enlargement of the conus heterogeneous enhancement.

A histological analysis of the specimens was performed. On hematoxylin and eosin (H\&E) stained section, areas of granulomatous inflammatory reaction and necrosis were observed (-Fig. 3). Areas suspicious for fungal organisms were visualized on sections. Capsulatum DNA was detected and prolonged fungal culture ultimately grew out Histoplasma capsulatum organisms. Final diagnosis was spinal cord compression caused by Histoplasma capsulatuma.

The patient received a specific antifungal therapy: itraconazole, $200 \mathrm{mg}$ per day for 6 months. She also has functional and sphincter rehabilitation. She was given a checkup 8 months later and we noticed a sensory and motor recovery with a muscular side at $4+/ 5$, autonomous walking was possible but somewhat difficult with sphincter control. Two years after the end of treatment, the patient was autonomous.

\section{Synthesis of Literature Dealing with Spinal Cord Compression Caused by Histoplasmosis $3,4,6-13$}

Adding our case to the literature review, there are 10 cases of histoplasmosis spinal cord compression reported. Seven

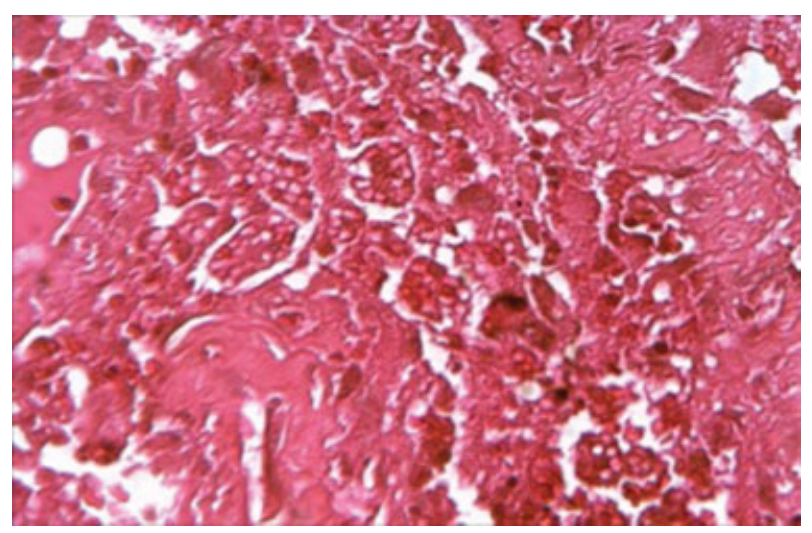

Fig. 3 Histoplasmosis granuloma due to Histoplasma capsulatum small yeast intra- and extrastrateum $\mathrm{H} \& \mathrm{E} \times 400$. 
Spinal Cord Compression Caused by Histoplasma capsulatum N'Dri-Oka et al. 111

\begin{tabular}{|c|c|c|c|c|c|c|c|c|c|c|}
\hline 总 & 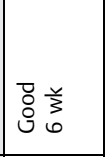 & & 总 & $\begin{array}{l}\square \\
\\
\end{array}$ & 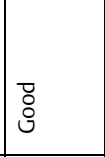 & 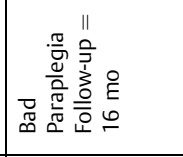 & 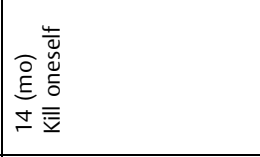 & 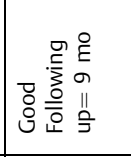 & 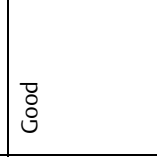 & 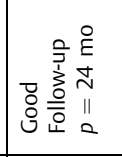 \\
\hline 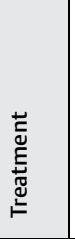 & 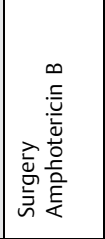 & & 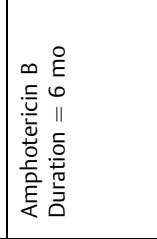 & 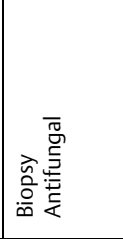 & 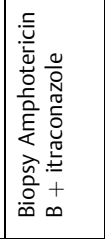 & 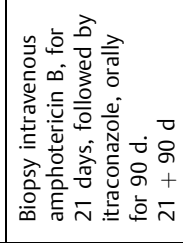 & 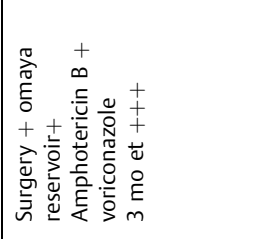 & 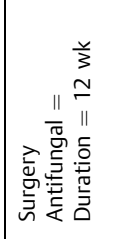 & 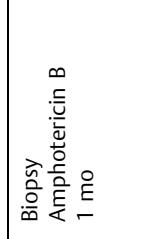 & 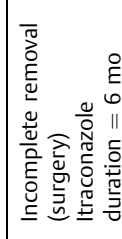 \\
\hline 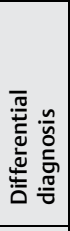 & & & 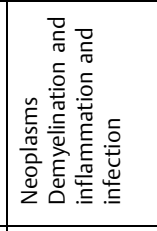 & 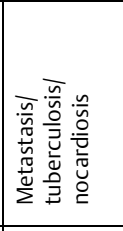 & 1 & & & \begin{tabular}{|l} 
言 \\
王
\end{tabular} & I & $\mid \begin{array}{l}\text { 高 } \\
\text { 互 }\end{array}$ \\
\hline 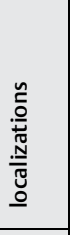 & 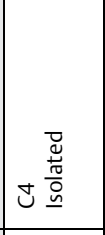 & 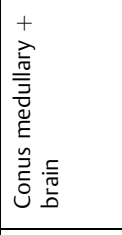 & 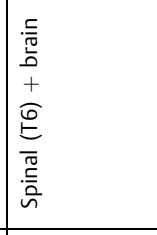 & 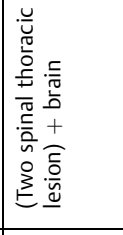 & 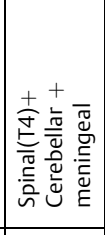 & 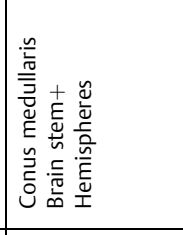 & 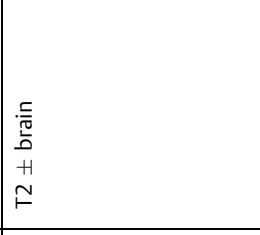 & 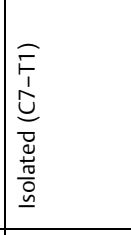 & 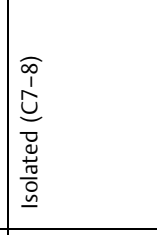 & 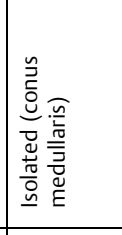 \\
\hline 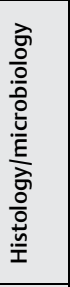 & 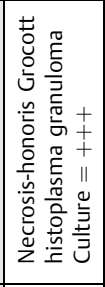 & 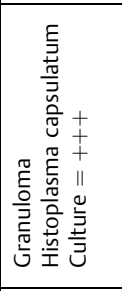 & 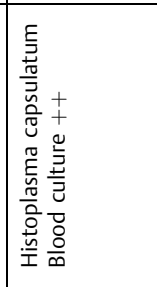 & 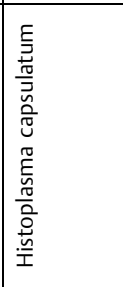 & 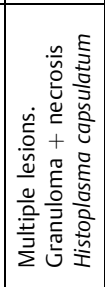 & 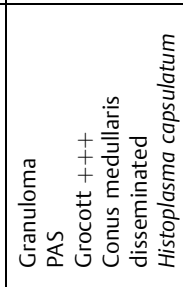 & 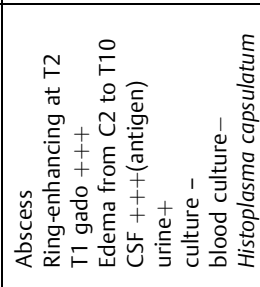 & 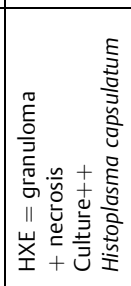 & 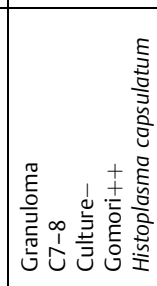 & 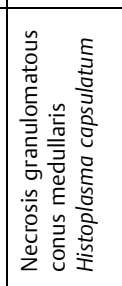 \\
\hline 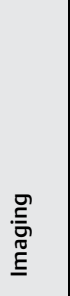 & 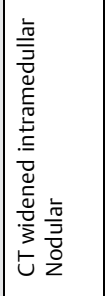 & 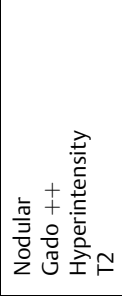 & 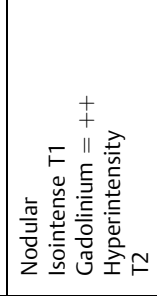 & 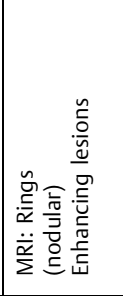 & 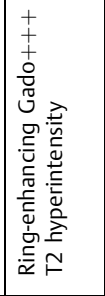 & 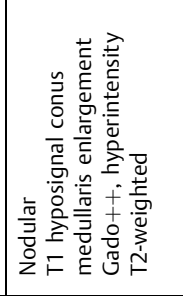 & 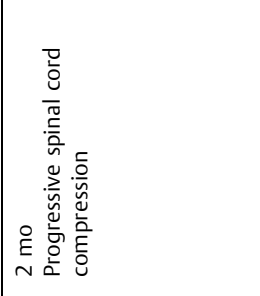 & 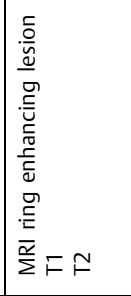 & 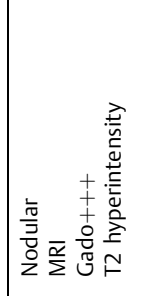 & 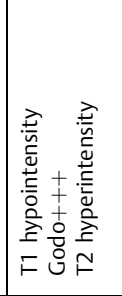 \\
\hline $\begin{array}{l}\frac{y}{\underline{\underline{E}}} \\
\underline{\underline{E}}\end{array}$ & 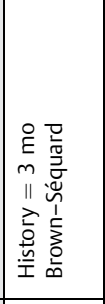 & 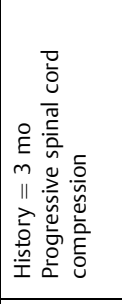 & 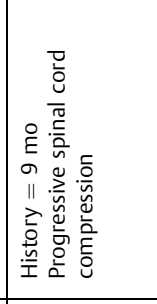 & 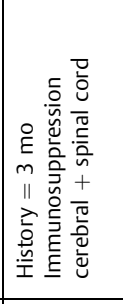 & 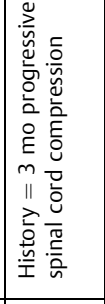 & 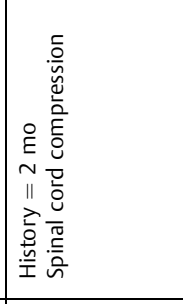 & 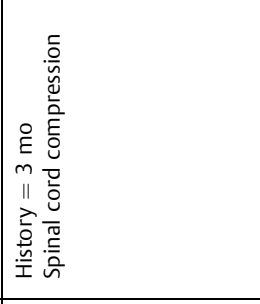 & 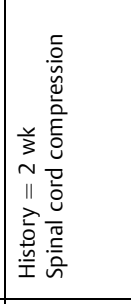 & 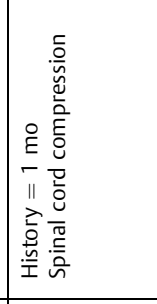 & 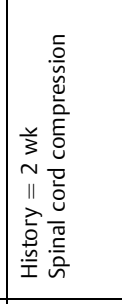 \\
\hline 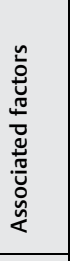 & 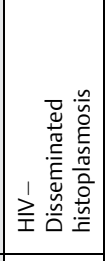 & & 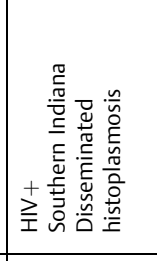 & 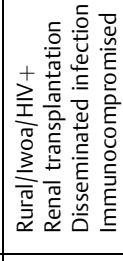 & 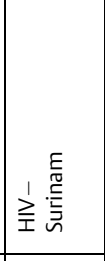 & 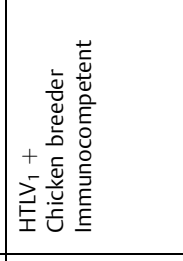 & 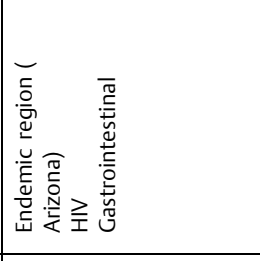 & 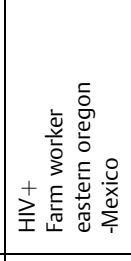 & 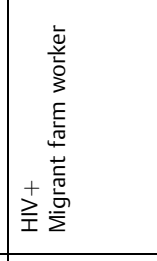 & 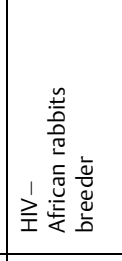 \\
\hline 爻 & $\frac{\infty}{\Sigma}$ & $\frac{n}{\Sigma}$ & $\frac{9}{4}$ & $\frac{n}{\Sigma}$ & $\frac{6}{\Sigma}$ & $\frac{8}{2}$ & $\mid \frac{7}{4}$ & $\frac{\hat{N}}{\bar{\Sigma}}$ & $\frac{\hat{N}}{\Sigma}$ & $\frac{f}{4}$ \\
\hline 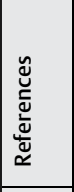 & 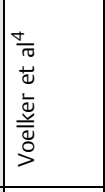 & 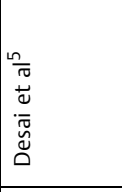 & 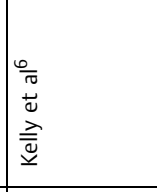 & 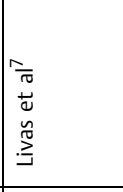 & 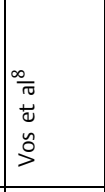 & 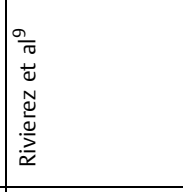 & 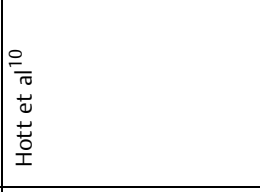 & 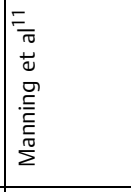 & 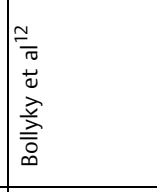 & 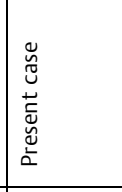 \\
\hline$z$ & - & N & m & o & in & 0 & $\wedge$ & $\infty$ & $a$ & 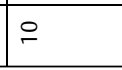 \\
\hline
\end{tabular}


patients came from endemic regions; one case from Arizona which is a nonendemic region, and in two cases the origin is not precised (-Table $\mathbf{1}$ ).

The mean age of the patient suffering from spinal cord compression caused by intramedullary histoplasmosis because of the Histoplasma capsulatum was 40 years old whose extremes are 16 and 75 years.

Histoplasmosis spinal cord compression history started progressively around 3 months in three cases. Spinal cord compression caused by histoplasmosis occurred in immunocompromised patient as positive HIV patient (four cases), $\operatorname{HTLV}_{1}$ (one case), or organ transplantation (one case). Patient worked as rabbit or chicken breeders (two cases) and farm worker (three cases). Only one patient came from endemic areas. All cases are caused by Histoplasma capsulatum.

The history duration average was 3 months varying from 2 weeks to 9 months. Spinal cord compression occurred in contex of disseminated in three cases $(n=6)$, associated with brain localization in five cases or gastrointestinal in one case $(n=8)$. Spinal cord compression was isolated in five cases $(n=10)$.

MRI gadolinium enhanced nodular ring in all the cases hypointensity $\mathrm{T} 1$ with gadolinium and hyperintensity $\mathrm{T} 2$. In all cases reported in the literature, spinal cord compression is mainly because of the Histoplasma capsulatum. Anatomical forms reported were abscess in one case, necrosis granuloma in six cases, and nonevidence myelitis was diagnosed in seven cases.

Differential diagnosis were tumors (primitive or metastasis) in four cases and in one case, it was demyelinating and inflammatory and infection disease (nocardiosis or tuberculosis) $(n=5)$.

Histological examination findings were necrotizing granuloma in seven cases, abscess in one case, and no precision in two cases $(n=10)$. Intramedullary spinal cord caused by Histoplasma capsulatum diagnosis was made after surgical removal in four cases, biopsy in five cases, and blood culture in only one case.

The patients felt better after an antifungal therapy in nine cases. In one case, treatment and outcomes were not precised. Antifungal were amphotericin B, itraconazole, or voriconazole. Association of these antifungal medicines was used in three cases and one antifungal medicine in four cases $(n=7)$. Median duration of antifungal therapy was 3 months (from 1 to 6 months; $n=4$ ). Follow-up was from 6 weeks to 24 months with a mean of 13 months and the outcome was good in seven cases, bad in one case, the patient kills oneself in one case, and nonprecised in one case.

\section{Discussion}

The severity of the neurologic presentation, lack of systemic signs, and extensiveness of central nervous system lesions on MRI are exceptional. To our knowledge, this is the third case of isolated spinal conus compression caused by histoplasmosis reported in non-immunocompromised host.
This study allows us to deal with epidemiology, clinics management, and outcome of such spinal cord compression.

Primary neurologic presentation of histoplasmosis is extremely rare, especially in young, non-immunocompromised hosts. A case of disseminated histoplasmosis in a 72-year-old man was reported, it was the first report of histoplasmosis presenting as a myelopathy. ${ }^{14}$

Spinal cord compression caused by histoplasmosis is high in the patient with history of organ transplantation, ${ }^{8}$ animal breeders, ${ }^{10}$ farm workers, ${ }^{12,13}$ endemic areas, ${ }^{7,9}$ and human immunodeficiency virus infection., ${ }^{72,13}$ It was intra-spinal cord abscess. ${ }^{6,8,11}$ Histoplasmosis in Histoplasma capsulatum is cosmopolitan. ${ }^{6,8}$ The predominance is male and the age is around the second decade of life. The initial lesion would be lung with an incubation period of 2 weeks, and then secondarily would be hematogenous dissemination., ${ }^{6,7}$ Professional and geographic origin seem to be predisposing factors. ${ }^{10,12,13}$

Even though histoplasmosis is endemic in our region, central nervous system localization is rare and especially for spinal localization, generally, the disease is observed in disseminated forms ${ }^{9}$ or associated with immunodeficiency. ${ }^{8,10,12,13}$ Brain localization and necrotizing granulomas are well known, ${ }^{8}$ but spinal cord compression caused by histoplasmosis is exceptional: only four isolated cases have been evaluated by MRI. ${ }^{6,12,13}$

Histoplasma capsulatum endemic areas are more extended than the one previously described. ${ }^{2}$ In fact, the case was reported in Arizona in host. ${ }^{11}$

Diagnosis must be evoked in patient with predisposing conditions such as endemic areas living, immunocompromised, rural people, animal breeders, and farm workers.

MRI showed enlargement of the spinal cord, with a lowintensity signal on T1-weighted images, high-intensity signal on T2-weighted images, and areas of intramedullar contrast enhancement. Because of the association of brain localization, it is mandatory to search for it when spinal cord location is diagnosed. The lesion is nodular or like a ring one or multiple granuloma. ${ }^{9}$ Conclusion of this review is that typically imaging presentation of intramedullary histoplasmosis is nodular or ring enhanced, isolated or multiple hypointensity T1 and hyperintensity T2.

Histopathological examination is also an evidence of the diagnosis of histoplasmosis. In the case of Histoplasma capsulatum, histopathological examination shows the presence of major forms of yeast (8-15 $\mathrm{m}$ diameter). Areas for fungal microorganisms were suspicious or visualized by H\&E section stain and Gomori Methenamine stain fungal element became clearly apparent. ${ }^{12}$ On the basis of the histopathology, they are of the following three forms: granuloma, ${ }^{3}$ abcess, ${ }^{11}$ and myelitis. In the literature, less than $50 \%$ of the cases were disseminated histoplasmosis and this can manifest itself by lymphocytic meningitis, focal cerebral or spinal lesions like in our case. Mycological data assessment given by direct examination with culture isolates microorganism. The polymerase chain reaction is useful for the diagnosis. A biopsy of the lesion showed macrophages containing yeast cells, with periodic acid-schiff test and Grocott staining aspects compatible with the presence of 
Histoplasma capsulatum. Other examinations such as polysaccharide may be contributory to histoplasma antigen detection in the serological test.

The current antifungal treatment recommended in violations of the central nervous system with molecule of choice is of course itraconazole. In our case, spinal cord improved with surgery associated to antifungus medication. Sometimes specific medical treatment alone is enough but with sufficient dosage. ${ }^{7}$ Successful medical treatment of a spinal cord because of the histoplasmosis after biopsy is reported again. ${ }^{7}$ In fact, antifungal therapy is the most important in the treatment. Because of neurological complications, we emphasize only biopsy and antifungal treatment. We think that the role of surgery is to get specimens for the diagnosis.

\section{Conclusion}

In endemic areas, histoplasmosis must be evoked as etiology of spinal cord compression. Cerebral imaging must be systematically performed because of the frequent association to brain localization.

\section{Conflict of Interest}

This article does not have any conflict of interest.

\section{References}

1 Trofa D, Nosanchuk JD. Histoplasmosis of the central nervous system. J Neuroparasitology 2012;3:1-7

2 Ajello L. Relationship Histoplasma capsulatum Avian habitats. Public Health Rep 1964;79:266-270
3 Bazan C III, New PZ; BazanC. Intramedullary spinal histoplasmosis efficacy of gadolinium enhancement. Neuroradiology 1991;33(2):190

4 Desai SP, Bazan C III, Hummell W, Jinkins JR. Disseminated CNS histoplasmosis. AJNR Am J Neuroradiol 1991;12(2):290-292

5 Jardim E, Takayanagui OM. Myelopathy caused by histoplasmosis: report of a case [in Portuguese]. Arq Neuropsiquiatr 1981;39(1): 115-118

6 Voelker JL, Muller J, Worth RM. Intramedullary spinal Histoplasma granuloma. Case report. J Neurosurg 1989;70(6): 959-961

7 Kelly DR, Smith CD, McQuillen MP. Successful medical treatment of a spinal histoplasmoma. J Neuroimaging 1994;4(4):237-239

8 Livas IC, Nechay PS, Nauseef WM. Clinical evidence of spinal and cerebral histoplasmosis twenty years after renal transplantation. Clin Infect Dis 1995;20(3):692-695

9 Vos MJ, Debets-Ossenkopp YJ, Claessen FAP, Hazenberg GJ, Heimans JJ. Cerebellar and medullar histoplasmosis. Neurology 2000;54(7):1441

10 Rivierez M, Heyman D, Brebion A, Landau-Ossondo M, Desbois N, Vally P. [Spinal cord histoplasmoma. A case report]. Neurochirurgie 2002;48(1):44-48

11 Hott JS, Horn E, Sonntag VK, Coons SW, Shetter A. Intramedullary histoplasmosis spinal cord abscess in a nonendemic region: case report and review of the literature. J Spinal Disord Tech 2003; 16(2):212-215

12 Manning TC, Born D, Tredway TL. Spinal intramedullary histoplasmosis as the initial presentation of human immunodeficiency virus infection: case report. Neurosurgery 2006;59(5):E1146, discussion E1146

13 Bollyky PL, Czartoski TJ, Limaye A. Histoplasmosis presenting as an isolated spinal cord lesion. Arch Neurol 2006;63(12): 1802-1803

14 Tan V, Wilkins P, Badve S, et al. Histoplasmosis of the central nervous system. J Neurol Neurosurg Psychiatry 1992;55(7): 619-622 